\title{
Preclinical research: a rise or dawn
}

\begin{abstract}
Drug discoveries and drug testing correspond to be the most vital areas of Pharmaceutical industries to invariably contribute medicine. Advanced research is prolonged right from the Phase I studies to carefully estimate the potential benefits of the specific drug. No compromise is attempted in the release of a medicine as examinations related to adverse reactions are carefully analyzed. Suitable preclinical studies are of major importance in validating the beneficial effects of a drug prior to the initializing of clinical trial. Though journals have been mentioning about bypassing preclinical trials with lower or mild dose to desirable subjects which directly derives conclusive evidences, it is more concerned with the regulatory channels to approve such studies. However, some reports have shown absence of probable correlation with higher animals and there have also been poor prognostic benefits after it has been released as a drug of choice for certain ailments. With strong preclinical background, it is worth to analyze wherein the lapse is as there are still unavailable drugs for various diseases. Approach towards drug discovery and drug testing thus has been a challenging concept in order to launch an efficient drug formulated for the specified conditions. How far it is challenging in the case of preclinical research is our ultimate concern for the current review. This review also focuses on the lapses that preclinical research is facing along with the failed reciprocation in phase III studies generating the clear consideration of pathophysiological differences from animals to human.
\end{abstract}

Keywords: clinical trial, drug discoveries, drug testing, preclinical
Volume 6 Issue I - 2018

\author{
Baskar Subramani,' Sadananda Rao \\ Manjunath ${ }^{2}$ \\ 'Nichi-Asia Life Sdn Bhd., Malaysia \\ ${ }^{2}$ Hygieia Therapeutics, Malaysia
}

Correspondence: Sadananda Rao Manjunath, Immunology Unit, Lot IG-2G Lanai Complex, No.2 Persiaran Seri Perdana, Precinct 10,62250 Wilayah Persekutuan Putrajaya, Malaysia,Tel +6036201 7969, Email smanjmicro@gmail.com

Received: November 20, 2017 | Published: January 09, 2018

\section{Introduction}

Complex processes are involved in developing a new drug along with its time and costs consumption. Prior to launching, it requires variety of evidences to show its potential activity. ${ }^{1}$ Key step in drug discovery is considered to be the target discovery which ranges from molecular entities to biological phenomenon. ${ }^{2}$ As there are urges in translating scientific discoveries into practical importance, preclinical studies using animal models play an important role in evaluating the efficacy and safety under clinical scenario. ${ }^{3}$ After enormous debates, it is concluded that the benefits of animal research is worth to manage public health and medical research. ${ }^{4}$ Subsequently, animal studies are justifiable if done in a way to minimize pain in animal models and wherever applicable alternative methods are explored and avoiding unnecessary cruel treatment to animals. ${ }^{5}$ However, various reports have enforced on the failures of trails that has never capitalized after thorough observation of safety and efficacy in animal models which mainly portraits the requirement of robustness in preclinical studies. ${ }^{6-10}$ For instance, reports say that, almost $95 \%$ of drugs that enter clinical trials related to stroke or septic shock do not get launched in the market though enormous expenditures have been dealt with the basic studies and drug discoveries ${ }^{11}$ and similarly, the average success rate in clinical cancer trials is less than $8 \%$ after preclinical estimation. ${ }^{10}$

Still it is recommended to circumvent preclinical studies because of the scientific, practical and ethical reasons, to conduct studies in humans, ${ }^{6}$ and thus prior to this attempt, preclinical drug combination studies in vitro and/or in animals has to be carried out to obtain the base for studies in human. ${ }^{12}$ Though the debate over use of the animals in medical experiments has a long and unpleasant history, eminent researches along with Nobel Prize winners have signed a declaration provided by the Research Defense Society has made claim that experiments on animals have made important contribution to advances in medicine and surgery. ${ }^{13}$ Research focused on pain research has shown enormous rate of failures and increased costs of drug development this is because of phenotypic differences and complexity in behavioral alterations along with the changes in daily activities and psychological disturbances. ${ }^{14,15}$ Moreover, the test and models of nociception has been questioned in the aspect of appropriateness between tests, models and procedures. ${ }^{16}$ Thus in order to develop robustness in pain management and to effectively gather required evidences, it is better to evaluate pain states in higher order preclinical species where we can predict whether biological mechanisms and specific compounds have relevance for clinical pain better than rodent species. The pain models in non-rodent species may enhance the speed, and in addition reduce costs along with the increased probable results for a successful analgesic development. ${ }^{17}$

\section{Hiccups in selection of animals, dose and formulations}

Arguments pertaining to the use of animals are strongly persisting ${ }^{5-19}$ mentioning the harmful and non therapeutic experimentation if wrong on humans, applies same to that of animals morally. ${ }^{20,21}$ This concerns to the reduced protection or care that an animal would receive and also the lack of certain characteristics that animals lack, appeals to their application for human requirements. ${ }^{22} \mathrm{An}$ ideal animal model is believed to be the one that matches with the specific study design with respect to anatomy and physiology. The disease setting has to closely resemble to that in human clinical situation. ${ }^{23}$ Concerns have been raised worldwide for the use of different animals such as Chimpanzee citing their lack of predictability and also claims of irrelevance for human behaviors are majorly under debate. ${ }^{24}$ Criticisms have been raised in the use of animal models has been summarized with the following errors of conception: ${ }^{25}$

a) Variations in dosing schedules and their schedules with uncertain relevance to the human condition 
b) Variations in the selection of animals, the methods of randomization, comparative therapy and losses in following ups.

c) Experimental groups with insufficient power, simple statistical analysis that has varied accounts of confusions

d) Lengths of follow up before the detection of disease outcome that doesn't correspond to the disease latency in humans.

Failures in animal models usually are because of disparities in disease specificity between the animal models and clinical trials that lead to inadequate data and incorrect conclusions on the outcomes. Thus there are critical parameters required to be monitored such as Sample size calculation, Monitoring of physiological parameters, eligibility criteria etc. have extensively shown bias in animal studies such as in acute ischemic stroke. ${ }^{26}$ Animal dosing need to be carefully considered in order to optimize the delivered agent to prevent or minimize adverse reactions and the vehicle selected to deliver substances also has to be on the length of the study and the nature of the substance being injected or administered. Along with dose optimization, route of administration also plays an important role. It requires the members to be aware of reasons for appropriate selection of the routes for administration. There are other critical issues also associated with the route selection such as volume of administration, site of delivery, $\mathrm{pH}$ of the substance and certain other factors also are majorly to be considered. Improper selection of any of these factors may result in bias and unintentional adverse effects on the experimental models and unreliable results. ${ }^{27}$

\section{Preclinical studies overall scenario}

Results of animal biomedical experiments have vastly failed to translate into human clinical trials which are mostly attributed because of differences in the underlying biology between humans and animals to shortcomings in the experimental design or to bias in the reporting of results from animal studies. ${ }^{28}$ Animal studies have been commented widely to be methodologically weak ${ }^{29}$ which likely continues to provide the biological basis for epidemiological investigation but substantial improvement is needed in how it has to be conducted and synthesized to improve the predictability of animal studies for the human condition. Ideas for implementation of consensus practices and outcomes pertaining to clinical translation aren't clearly understood that can provide more comprehensive, transparent, evidence based and theoretically informed rationale for analysis of preclinical studies. ${ }^{30}$

With respect to clinical studies, it depends on the study protocols that are designed after evidential information generated from preclinical studies. The application of this data to the human studies is of major concern wherein the paramount importance lies with the ability to understand the preclinical study results before arriving at any conclusion. ${ }^{31}$ To comprehend this scenario, there were 300 negative studies published in selectively identified SCI indexed Indian Journals out of which only 19 studies had animal subjects. Majority of animal negative studies was found to be calculated without the inclusion of power and sample size. There are occurrences wherein under-reporting of various parameters was noticeable. Criticism was strongly raised on the Ethics Committee which was the reason for the reduction in sample size. ${ }^{32}$ Sample size is a serious consideration for animal studies as the less number of the animals would misinterpret the significance in the study and it would be wastage of resources along with ethical issues to deal with markedly more number of animals. ${ }^{33}$ Regarding the wastage of resources, it was remarked by
Mandal et al., ${ }^{34}$ that animal testing wastes time and resources by misleading researchers. Dr. Albert Sabin, who established Oral polio vaccine has stated in his testimony that his work had a delay because of the "erroneous conception of the nature of the human disease based on misleading experimental models of the disease in monkeys". There is one other testimony by Dr. Richard Klausner who has said that "the history of cancer research has been a history of curing cancer in the mouse. We have cured mice of cancer for decades and it simply didn't work in humans, we need to acknowledge the fact that use of animals will not make us better scientists, but bitter scientists".

\section{Justification of unwarranted results}

NIH has recently proposed to undertake multi-dimensional approach to mention the dependency on male cells and animals in preclinical research. Policies to improve sex balance in research are being vastly discussed. ${ }^{35}$ Evidences suggest that most of the discoveries have not stood the test of time. Reproducibility has been a major concern in both basic and preclinical research where it is noted that good scientific practice are not considered. ${ }^{36}$ To criticize animal studies, Pound et al has reported that clinical trials of nimodipine and low level laser therapy when conducted concurrently with the animal studies, clinical studies of fluid resuscitation, thrombolytic therapy and endothelin receptor blockade went on though evidence was available of harm from the animal studies. It thus suggests that, the animal data generated is irrelevant regardless to the requirement of animal studies prior to clinical trials. ${ }^{37}$

Mice have taken the blame as being most uncomfortable truths in preclinical research. More than $80 \%$ therapeutics has failed after animal studies in which it was suggested as safe and effective. Thus animal studies are criticized to be poor predictors of experimental drugs. ${ }^{38}$ Even rats of same strain when purchased from different suppliers have produced different test results; more specifically, when 12 different behavioral measures of pain sensitivity which are important markers of spinal cord injury was found to produce variable responses in 11 strains of mice. There was no proper patterns of predictions how the strains would respond. ${ }^{39}$ It is widely known and accepted that animal toxicology studies needs to contain modern concepts of pharmacokinetics and toxicokinetics. The method of action studies should also determine whether animal data can be used for determining risk in human subjects. The parameters applied for environmental toxicants in animal studies such as threshold exposures, maximum permissible exposures and toxic effects that were estimated has to be interpreted with caution when accounted on human. Epidemiology studies that are well-performed are considered to be best suitable for determining human risk and effects of environmental toxicants. ${ }^{40}$ Presently, measures are being taken to find effective alternatives to animal studies with the use of cell and tissue culture and computer simulation. ${ }^{41}$

\section{Regulatory perspectives of studies using ani- mals}

With the controversies upon preclinical research still existing, guidelines to conduct quality research with respect to animal utilization criteria are clearly being demonstrated in the International Council on Harmonization of Technical Requirements for Registration of Pharmaceuticals for Human Use (ICH), ${ }^{42}$ Central Drugs standard Control Organization (Schedule Y), ${ }^{43}$ The Organization for Economic Co-operation and Development (OECD). ${ }^{44}$ These guidelines have 
been promoted to improve the standards of well-being of people around the world. The studies conducted are being thoroughly monitored and performed as per the guidelines. The guidelines have also given reporting formats in a consolidated manner that could produce consistency ${ }^{45}$ Apart from this, there are articles to support the calculation of sample size which is a vital component of design of any research using animals. ${ }^{32}$ Similarly there are published articles to provide ideas on the design and statistical analysis of experiments using laboratory animals. ${ }^{46}$ These guidelines not only limit the mistreatment of animals but also protect the groups and public for proper care of animal research. ${ }^{47}$

\section{Acknowledgements}

None.

\section{Conflict of interest}

Author declares that there is no conflict of interest.

\section{References}

1. Hughes JP, Rees S, Kalindjian SB, et al. Principles of early drug discovery. Br J Pharmacol. 2011;162(6):1239-1249.

2. Yang Y, Adelstein SJ, Kassis AI. Target discovery from data mining approaches. Drug Discov Today. 2009;14(3-4):147-154.

3. Suzuki Y, Yeung AC, Ikeno F. The pre-clinical animal model in the translational research of interventional cardiology. JACC Cardiovasc Interv. 2009;2(5):373-383.

4. Festing S, Wilkinson R. The ethics of animal research. Talking Point on the use of animals in scientific research. EMBO Rep. 2007;8(6):526-530.

5. Gannon F. Animal rights, human wrongs? Introduction to the Talking Point on the use of animals in scientific research. EMBO Rep. 2007;8(6):519-520.

6. Dyson A, Singer M. Animal models of sepsis: why does preclinical efficacy fail to translate to the clinical setting?. Crit Care Med. 2009;37(1 Suppl):S30-S37.

7. Neuhaus AA, Rabie T, Sutherland BA, et al. Importance of preclinical research in the development of neuroprotective strategies for ischemic stroke. JAMA Neurol. 2014;71(5):634-639.

8. Kimmelman J, Moqul JS, Dirnaql U. Distinguishing between exploratory and confirmatory preclinical research will improve translation. PLoS Biol. 2014;12(5):e1001863.

9. Lowenstein PR, Castro MG. Uncertainty in the translation of preclinical experiments to clinical trials. Why do most phase III clinical trials fail? Curr Gene Ther. 2009;9(5):368-374.

10. Mak IW, Evaniew N, Ghert M. Lost in translation: animal models and clinical trial in cancer treatment. Am J Transl Res. 2014;6(2):114-118.

11. Hartung T. Look back in anger- what clinical studies tell us about preclinical work. ALTEX. 2013;30(3):275-291.

12. Chou TC. Preclinical versus clinical drug combination studies. Leuk Lymphoma. 2008;49(11):2059-2080.

13. Matthews RA. Medical progress depends on animal models- doesn't it? $J$ R Soc Med. 2008;101(2):95-98.

14. King T, Porreca F. Preclinical assessment of pain: improving models in discovery research. Curr Top Behav Neurosci. 2014;20:101-120.

15. Cobos EJ, Portillo-Salido E. "Bedside-to-Bench" Behavioral Outcomes in Animal Models of Pain: Beyond the Evaluation of Reflexes. Curr Neuropharmacol. 2013;11(6):560-591.
16. Barrot M. Tests and models of nociception and pain in rodents. Neuroscience. 2012;211:39-50.

17. Henze DA, Urban MO. Large Animal Models for Pain Therapeutic Development. USA: (Chapter 17) CRC Press/Taylor \& Francis; 2010.

18. Olsson IA, Sandoe P. "What's wrong with my monkey?" Ethical perspectives on germline transgenesis in marmosets. Transgenic Res 2010;19(2):181-186.

19. Greek R, Shanks N. Complex systems, evolution, and animal models. Stud Hist Philos Biol Biomed Sci. 2011;42(4):542-544.

20. Nobis N. The harmful, nontherapeutic use of animals in research is morally wrong. Am J Med Sci. 2011;342(4):297-304.

21. Pluhar EB. Experimentation on humans and nonhumans. Theor Med Bioeth. 2006;27(4):333-355.

22. Walker RL. Human and animal subjects of research: the moral significance of respect versus welfare. Theor Med Bioeth. 2006;27(4):305-331.

23. Saraf SK, Kumaraswamy V. Basic research: Issues with animal experimentations. Indian J Orthop. 2013;47(1):6-9.

24. Shanks N, Greek R, Greek J. Are animal models predictive for humans?. Philos Ethics Humanit Med. 2009;4:2.

25. Greek R, Menache A. Systemic reviews of animal models: methodology versus epistemology. Int J Med Sci. 2013;10(3):206-221.

26. Van Der Worp HB, Howells DW, Sena ES, et al. Can animal models of disease reliability inform human studies?. PLoS Med. 2010;7(3):e1000245.

27. Turner PV, Brabb T, Pekow C, et al. Administration of substances to laboratory animals: routes of administration and factors to consider. $J \mathrm{Am}$ Assoc Lab Anim Sci. 2011;50(5):600-613.

28. Tsilidis KK, Panagiotou OA, Sena ES, et al. Evaluation of excess significance bias in animal studies of neurological diseases. PLoS Biol. 2013;11(7):e1001609.

29. Bracken MB. Why are so many epidemiology associations inflated or wrong? Does poorly conducted animal research suggest implausible hypotheses?. Ann Epidemiol. 2009;19(3):220-224.

30. Henderson CV, Kimmelman J, Fergusson D, et al. Threats to validity in the Design and Conduct of Preclinical Efficacy Studies: A Systematic Review of Guidelines for In Vivo Animal Experiments. PLoS Med. 2013;10(7):e1001489.

31. Ramkumar A. Early phase studies in India: Are we too early to explore? Indian J Pharmacol. 2008;40(5):189-190.

32. Charan J, Saxena D. Negative animal studies published in Indian Medical Journal: Are they methodologically strong enough to conclude what they are concluding? J Pharm Bioallied Sci. 2014;6(2):134-136.

33. Charan J, Kantharia ND. How to calculate sample size in animal studies? J Pharmacol Pharmacother. 2013;4(4):303-306.

34. Mandal J, Parija SC. Ethics of involving animals in research. Trop Parasitol. $2013 ; 3(1): 4-6$.

35. Mc Cullough LD, De Vries GJ, Miller VM, et al. NIH initiative to balance sex of animals in preclinical studies: generative questions to guide policy, implementation, and metrics. Biol Sex Differ. 2014;5:15.

36. Begley CG, Loannidis JP. Reproducibility in Science: improving the standard for basic and preclinical research. Circ Res. 2015;116(1):116-126.

37. Pound P, Ebrahim S, Sandercock P, et al. Where is the evidence that animal research benefits humans? BMJ. 2004;328(7438):514-517.

38. Perrin S. Preclinical research: Make mouse studies work. Nature. 2014;507(7493):423-425. 
39. Akhtar A. The flaws and human harms of animal experimentation. Camb $Q$ Healthc Ethics. 2015;24(4):407-419.

40. Brent RL. Utilization of animal studies to determine the effects and human risks of environmental toxicants (drugs, chemicals, and physical agents). Pediatrics. 2004;113(4 Suppl):984-995.

41. Kehinde EO. They see a rat, we seek a cure for diseases: the current status of animal experimentation in medical practice. Med Princ Pract. 2013;22(Suppl 1):S52-S61.

42. ICH Guidelines. ICH: Harmonization for better health. Switzerland; 2005.

43. Requirements and guidelines for permission to import and/or manufacture of new drugs for sale or to undertake clinical trails. Drugs and cosmetics. 1945:1-46.
44. OECD Guidelines for the Testing of Chemicals. OCED.org, France; 2012.

45. Kilkenny C, Browne WJ, Cuthill IC, et al. Animal research: Reporting in vivo experiments: The ARRIVE guidelines. Br J Pharmacol. 2010;160(7):1577-1579.

46. Festing MF, Altman DG. Guidelines for the design and statistical analysis of experiments using laboratory animals. ILAR J. 2002;43(4):244-258.

47. National Research Council (US) Committee. Science, Medicine, and Animals. USA: National Academies Press; 2004:1-52. 